\title{
A REMARK CONCERNING A THEOREM OF B. FRIEDMAN
}

\section{J. FELDMAN}

In [1], the following theorem is stated: let $T$ be a densely defined linear operator with closed range in the Hilbert space $\mathfrak{H C}$, with a densely defined adjoint $T^{*}$ also having closed range. Let $\phi, \psi$ be vectors in $\mathfrak{H}$, and let $\phi \otimes \psi$ be the operator defined by $\phi \otimes \psi(x)$ $=(x, \phi) \psi$. Then $T+\phi \otimes \psi$ also has closed range.

Of course, the fact that $T^{*}$ is densely defined implies that $T$ is pre-closed; but an examination of the proof shows that it actually requires that $T$ be a closed operator. Under this assumption, a simpler proof can be given; and the need for some such condition will be shown by example.

TheOREm. Let $T$ be a closed, densely defined operator with closed range. Then $S=T+\phi \otimes \psi$ also has a closed range.

Proof. The nullspace $\mathfrak{I}_{T}$ of a closed operator $T$ is closed, and its domain $\mathscr{D}_{T}$ is the sum of the two subspaces $\mathfrak{N}_{T}$ and $\mathscr{D}_{T} \cap \mathfrak{T}_{T}^{\frac{1}{T}}=S_{T}$, since $x$ in $D_{T}$ can be written $\left(x-P_{\mathscr{Y}_{T}} x\right)+P_{\mathscr{T}_{T}} x$ (where $P_{\mathfrak{T}}$ denotes the orthogonal projection on the subspace $\mathfrak{T})$. If we use the graph norm on $\mathfrak{D}_{T}$, given by the inner product $\langle x, y\rangle=(x, y)+(T x, T y)$, then $\mathfrak{N}_{T}$ and $S_{T}$ are complete, and $\mathscr{D}_{T}$ is their Hilbert space direct sum.

$T$ restricted to $\delta_{T}$ is a 1-1 continuous operator from $S_{T}$ (in the graph norm) to the range $R_{T}$ of $T$. The closed graph theorem tells us that its inverse $R$ is continuous, as an operator from the Hilbert space $R_{T}$ to $S_{T}$. Now, the orthogonal complement $[\phi]^{\perp}$ of $\phi$ in $\mathcal{T C}$ is closed in $\mathcal{T}$. Thus its intersection with $\delta_{T}$ is closed in the graph norm. Then $R^{-1}\left([\phi]^{\perp} \cap S_{T}\right)=T\left([\phi]^{\perp} \cap S_{T}\right)$ isclosed in $\mathcal{H C} . T\left([\phi]^{\perp} \cap S_{T}\right)=S\left([\phi]^{\perp} \cap S_{T}\right)$ $\subset \mathfrak{R}_{S} \subset \mathfrak{R}_{T}+[\psi]=T\left(\mathcal{S}_{T}\right)+[\psi]$. Now, the codimension of $T\left([\phi]^{\perp} \cap S_{T}\right)$ in $T\left(\mathcal{S}_{T}\right)+[\psi]$ is at most two, so that of $T\left([\phi]^{\perp} \cap S_{T}\right)$ in $R_{s}$ is again at most two. Since $T\left([\phi]^{\perp} \cap \mathcal{S}_{T}\right)$ is closed, $R_{s}$ is also closed.

REMARK 1 . If $T$ had been merely preclosed, but with closed range, then it is easy to see $R_{\bar{T}}=R_{T}$, so that $\bar{S}=\bar{T}+\phi \otimes \psi$ has closed range.

REMARK 2. Here is an example of an operator $T$ which is densely defined, bounded, and has closed range, and whose adjoint $T^{*}$ is therefore bounded and has closed range, but for which $S=T+\phi \otimes \psi$ will not have closed range, for certain $\phi$ and $\psi$.

Let $\mathfrak{K}_{0}$ be a proper infinite-dimensional subspace of $\mathfrak{H}$, and

Received by the editors December 23, 1957. 
$\psi, \psi_{1}, \psi_{2}, \psi_{3}, \cdots \quad$ an orthonormal basis for $\mathcal{H}_{0}$. Let $\psi_{n}^{\prime}$ $=n^{-1 / 2}\left((n-1)^{1 / 2} \psi+\psi_{n}\right)$. Then the set $\Psi=\left\{\psi, \psi_{1}^{\prime}, \psi_{2}^{\prime}, \cdots\right\}$ is linearly independent, and $\left\|\psi_{n}^{\prime}-\psi\right\|^{2} \rightarrow 0$. Enlarge $\Psi$ to a maximal linearly independent set $\Phi$ in $\mathcal{F}_{0}$. Thus, the linear combinations of elements of $\Phi$ span $\mathfrak{K}_{0}$. Let $\mathfrak{K}_{0}$ be the set of all linear combinations of elements of $\Phi-\{\psi\}$. Then $\mathcal{K}_{0}$ has the following properties:

(1) $\mathfrak{K}_{0}$ is dense in $\mathfrak{K}_{0}$.

(2) $\psi \notin \varkappa_{0}$.

(3) $\mathfrak{K}_{0}+[\psi]=\mathfrak{K}_{0}$.

Let $\phi$ be any unit vector in $\mathcal{F}_{0}^{\perp}$. Let $T$ be the restriction of $P_{\mathcal{H}}-\phi \otimes \psi$ to $\mathfrak{K}_{0}+\mathfrak{H}_{0}^{\perp}$. Then clearly $\mathfrak{R}_{T} \subset \mathfrak{H}_{0}$. Further, $T\left|\mathfrak{K}_{0}=P_{\mathfrak{K}_{0}}\right| \mathfrak{K}_{0}$, so $\mathfrak{K}_{0} \subset \mathfrak{R}_{T}$. Finally, $T \phi=-\psi$, so $\mathcal{R}_{T}=\mathfrak{H}_{0}$. Notice also that $T^{*}=P \mathcal{F}_{0}$ $-\psi \otimes \phi$, and so if $x=x_{1}+x_{2}+\alpha \psi$, where $x_{1} \in \mathcal{H}_{0} \cap[\psi]^{\perp}, x_{2} \in \mathcal{H}_{0}^{\perp}$, then $T^{*} x=x_{1}+\alpha(\psi-\phi)$. Thus $\mathfrak{R}_{T}^{*}=\left(\mathfrak{F C}_{0} \cap[\psi]^{\perp}\right)+[\psi-\phi]$, clearly closed. However, $T+\phi \otimes \psi=P \mathcal{K}_{0} \mid \mathfrak{K}_{0}$ has $\mathfrak{K}_{0}$ as its range.

\section{REFERENCE}

1. B. Friedman, Operations with a closed range, Comm. Pure Appl. Math. VIII, vol. 4 (1955) p. 539.

University of California at Berkeley 